\title{
Breakdown of elasticity in amorphous solids
}

\author{
Giulio Biroli ${ }^{1,2 \star}$ and Pierfrancesco Urbani ${ }^{1}$
}

\begin{abstract}
What characterizes a solid is the way that it responds to external stresses. Ordered solids, such as crystals, exhibit an elastic regime followed by a plastic regime, both understood microscopically in terms of lattice distortion and dislocations. For amorphous solids the situation is instead less clear, and the microscopic understanding of the response to deformation and stress is a very active research topic. Several studies have revealed that even in the elastic regime the response is very jerky at low temperature, resembling very much the response of disordered magnetic materials ${ }^{1-6}$. Here we show that in a very large class of amorphous solids this behaviour emerges upon decreasing temperature, as a phase transition, where standard elastic behaviour breaks down. At the transition all nonlinear elastic moduli diverge and standard elasticity theory no longer holds. Below the transition, the response to deformation becomes history- and time-dependent.
\end{abstract}

Our work connects two different lines of research on amorphous solids such as structural, colloidal and granular glasses. The first focuses on their behaviour at low temperature. With the aim of understanding the response of glasses to deformations, there have been extensive numerical studies of stress versus strain curves obtained by quenching model systems at zero temperature. One of the main outcomes is that the increase of the stress is punctuated by sudden drops related to avalanche-like rearrangements both before and after the yielding point ${ }^{1-6}$. This behaviour makes the measurements, and even the definition of elastic moduli fairly involved. In a series of works, Procaccia et al. have given evidence that in some models of glasses, such as Lennard-Jones mixtures (and variants), nonlinear elastic moduli exhibit diverging fluctuations, and linear elastic moduli differ depending on the way they are defined from the stress-strain curve $e^{7,8}$. Another independent research stream has focused on gaining an understanding of the jamming and glass transitions of hard spheres both from real-space and mean-field theory perspectives $^{9,10}$. The exact solution obtained in the limit of infinite dimensions revealed that by increasing the pressure a hard sphere glass exhibits a transition within the solid phase, where multiple arrangements emerge as different competing solid phases ${ }^{11,12}$. This is called the Gardner transition, in analogy with previous results in disordered spin models ${ }^{13,14}$. Recent simulations have confirmed that in three dimensions these different arrangements indeed become increasingly long-lived, possibly leading to ergodicity breaking ${ }^{15}$. These mean-field analyses complement and strengthen all the remarkable results found in the past two decades on jammed hard spheres glasses. The major outcome of these real-space studies was the discovery that amorphous jammed solids are marginally stable - that is, characterized by soft modes and critical behaviour, and in consequence by properties which are very different from those of usual crystalline solids ${ }^{16-20}$. In ref. 21 , it was argued that this is due to the way in which they are formed: unstable elementary excitations progressively rarify during a crunch, and jamming takes place exactly when no excitation is left, leaving the system solid but at the verge of instability (that is, marginally stable). Within mean-field theory, the results found at jamming are a consequence of a more general marginal stability: the appearance of multiple competing particle arrangements is accompanied by the emergence of long-range power-law correlations and soft modes. These are related to the Goldstone modes of the Gardner transition and are present not just at jamming - that is, at infinite pressure-but in the entire high-pressure regime beyond the critical Gardner point ${ }^{10,22}$.

Here we show that also models of structural glasses exhibit this transition when decreasing the temperature, and that this drastically affects their elastic behaviour. In particular, we reveal that elastic anomalies, such as the ones found in zero-temperature simulations, are a signature of this phase transition. To show the existence and the properties of the Gardner transition in structural glasses, we focus on a system of soft elastic spheres, which has been studied recently in several numerical simulations and shown to behave as canonical glass formers ${ }^{23-25}$. The interaction potential between particles reads

$$
\hat{V}_{\mathrm{HSS}}(r)=\frac{V_{0}}{2}\left(1-\frac{r}{\mathcal{D}}\right)^{2} \theta\left(1-\frac{r}{\mathcal{D}}\right)
$$

where $r$ is the distance between particles, $V_{0}$ is the interaction strength, $\mathcal{D}$ the interaction range and the $\theta$ function is the usual Heaviside step function. We choose this model since, in the limit $V_{0} \rightarrow \infty$, it maps on hard spheres with diameter $\mathcal{D}$. This enables us to make connections with previous results on jamming.

In this work we want to study the elastic properties of amorphous solids created by thermal quenches and also by compression. Theoretically, these solids are actually ultra-viscous liquids observed on timescales on which flow is absent. From the energy landscape perspective $^{26}$, these are systems unable to escape from a given metabasin within the experimental timescale. The large dimensional limit $(d \rightarrow \infty)$ is particularly useful to analyse these long-lived amorphous metastable states. Because the lifetime of metastable states diverges exponentially with $d$, one does not have to develop a full dynamical treatment, but can instead resort to a generalized thermodynamic framework able to capture the properties of metastable states ${ }^{27,28}$. What is generically considered a weakness of mean-field theory-the inability to describe activated dynamics in the supercooled regime-here becomes an advantage. In the infinite dimensional limit, metabasins become very long-lived below a welldefined temperature $T_{\mathrm{MCT}}$, corresponding to the mode coupling transition (MCT). Although in three dimensions the increase of the lifetime of metabasins is not as sharp below $T_{\mathrm{MCT}}$ (MCT becomes a crossover), in the experimentally relevant regime in which we are interested, amorphous solids do become well-defined metastable states. Indeed, for realistic quenches $\left(0.1-100 \mathrm{~K} \mathrm{~min}^{-1}\right)$, supercooled liquids fall out of equilibrium at a temperature $T_{\mathrm{g}}$ well below $T_{\mathrm{MCT}}$, and their properties do not change with time (except

${ }^{1}$ IPhT, Université Paris Saclay, CEA, CNRS, F-91191 Gif-sur-Yvette Cedex, France. ${ }^{2}$ Laboratoire de Physique Statistique, École Normale Supérieure, 24 rue Lhomond, 75005 Paris, France. *e-mail: giulio.biroli@cea.fr 


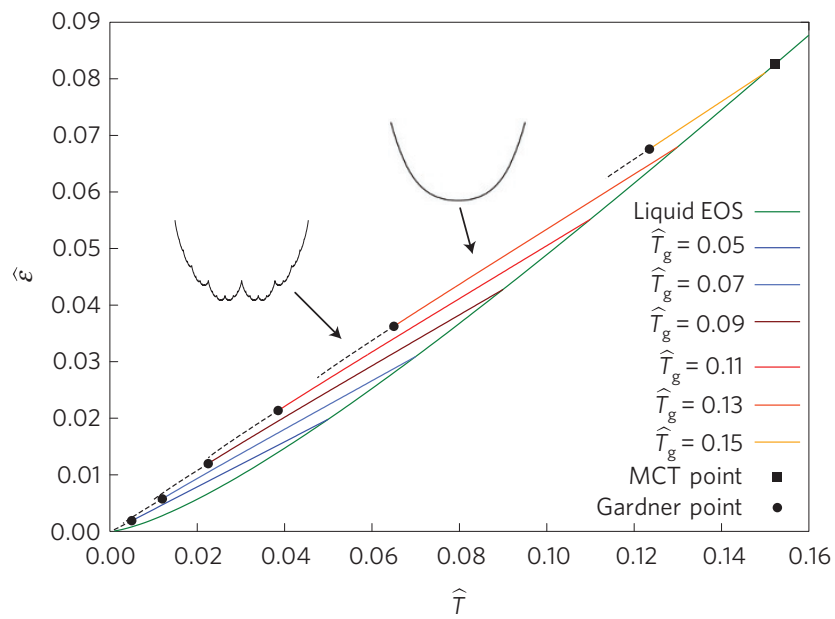

Figure 1 | Energy versus temperature (in rescaled units, see Supplementary Methods) for $\hat{\varphi}=\varphi 2^{d} / d=8$. EOS denotes the equilibrium line obtained by the equation of state. The other lines correspond to amorphous solids created by different cooling rates (slower to higher from bottom to top). Lines become dashed when the Gardner transition takes place. The change in the free-energy landscape at the Gardner transition is shown pictorially.

for very large times where ageing sets in); hence, the generalized thermodynamic framework ${ }^{27,28}$ we use is particularly well adapted.

To solve the model (1) in the $d \rightarrow \infty$ limit, and to study the properties of the metastable amorphous solids, we use the replica method, whose order parameter is the mean square displacement between couples of replicas $\left(x_{i}^{(a)}\right.$ is the position of the particle $i$ in replica $a$ ):

$$
\Delta_{a b}=\frac{d}{N \mathcal{D}^{2}} \sum_{i=1}^{N}\left|x_{i}^{(a)}-x_{i}^{(b)}\right|^{2}
$$

Roughly speaking, this order parameter allows one to study the statistical properties of the metabasins and probe their ruggedness: a breaking of replica symmetry means that that different replicas are trapped in different minima and, hence, that an ergodicity-breaking transition has taken place within the solid phase. Technically, the replica method intervenes because the metabasins in which the system is trapped play a role similar to quenched disorder in spin glasses: to obtain the average value of the elastic moduli, and their fluctuations, we need to use the replica method to handle the average over metabasins. This method has been developed and explained in full detail in several recent works on hard spheres ${ }^{29-31}$. Here we directly present our main results and refer to Supplementary Methods, in which the derivation and the generalization to compute the elastic moduli is presented.

Henceforth we consider packing fractions such that $T_{\mathrm{MCT}}(\varphi)>0$ and focus on glass states formed by slow quenches below it $\left(T_{\mathrm{MCT}}(\varphi)\right.$ rises from zero at a well-defined packing fraction $\varphi$, which in three dimensions should correspond to $\varphi_{\mathrm{MCT}} \simeq 0.58$ ). For a given cooling rate, the system follows the equilibrium line in the energytemperature plane until it falls out of equilibrium and becomes an amorphous solid at $T_{\mathrm{g}}$. We have computed both the equilibrium and the amorphous solid branches, as shown in Fig. 1 for a given packing fraction. The main result is that generically, by decreasing the temperature, amorphous solids undergo a Gardner transition at a temperature $T_{\mathrm{G}}(\varphi)$. The lower the glass transition temperature $T_{\mathrm{g}}$, the more one has to cool to reach $T_{\mathrm{G}}(\varphi)$. By comparing the results obtained for amorphous solids formed at the same $T_{\mathrm{g}}$ at different packing factions, we find that $T_{\mathrm{G}}(\varphi)$ decreases when $\varphi$ is increased, as shown in Fig. 2 (for too small densities, when $T_{\mathrm{g}}$ crosses $T_{\mathrm{MCT}}$, it is simply not possible to create a solid). We conclude this analysis by

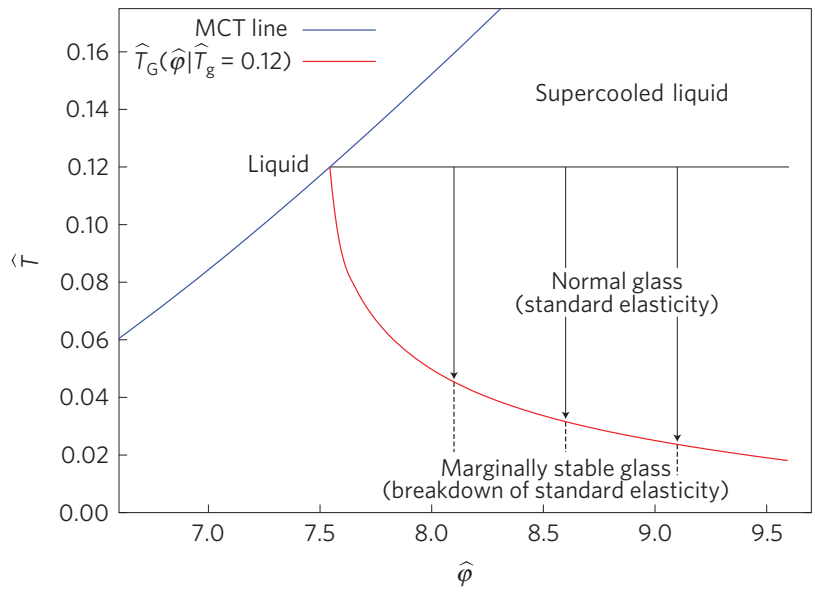

Figure 2 | Evolution of the Gardner transition temperature (red line) obtained by cooling amorphous solids at fixed $\varphi$. All amorphous solids are formed at the same $\widehat{T}_{g}=0.12$. The blue line denotes the MCT transition temperature as a function of $\widehat{\varphi}$.

considering glasses formed by compression to derive a relationship with studies on hard spheres and jamming. In this case, we find first a direct and then an inverse Gardner transition, as shown in Fig. 3. The results obtained for glasses formed at the same $\varphi_{\mathrm{g}}$ at different temperatures show that, the higher the temperature, the smaller is the extent of the Gardner phase, thus creating a dome in the $T-\varphi$ plane. (At very large $\varphi$, the glass state becomes unstable again. This new Gardner transition is completely disconnected from the jamming point.) This re-entrant behaviour is in agreement with very recent studies on the spectrum of harmonic vibrations of elastic sphere glasses $^{22,32}$.

In summary, we have shown that, by cooling, amorphous solids undergo the same transition towards a marginal glass state as found for hard spheres ${ }^{12}$. The marginality of this new solid phase is related to the presence of long-range correlations and soft modes ${ }^{10,22}$. We can now turn to our main concern, which is the change in the elastic properties of the solid approaching the Gardner transition. For a normal elastic solid, for example, a crystal, a small shear strain $\gamma$ induces a change in free energy per unit volume equal to

$$
\frac{\mathcal{F}_{\mathrm{el}}}{V}=\frac{\mu_{2}}{2} \gamma^{2}+\frac{\mu_{4}}{4 !} \gamma^{4}+\ldots
$$

where $V$ is the volume and $\mu_{n}$ is the $n$th order elastic modulus: $\mu_{n}=\left(\mathrm{d} \sigma^{n-1}\right) /\left(\mathrm{d}^{n-1} \gamma\right)$, where $\sigma$ is the stress $\left(\mu_{2}\right.$ is the usual linear shear modulus). This is also true for amorphous solids, but only above the Gardner transition. Our explicit computation in the limit of infinite dimensions shows that in this regime all elastic moduli are well defined (up to fluctuations of the order $1 / \sqrt{V}$ ) and that they depend on the glass state only through the value of $T_{\mathrm{g}}$-that is, the speed of the quench used to form the glass-and of the applied temperature and packing fraction. (We are focusing on the NVT ensemble, but of course our results can be translated to other cases-for example, the more experimentally relevant NPT case.) The situation changes drastically approaching the Gardner transition line, at which the fluctuations of all elastic moduli blow up. Although averages remain featureless, the fluctuations from one glass state to another, rescaled by their typical value $1 / \sqrt{V}$, diverge as:

$$
\overline{\left(\delta \mu_{n} \sqrt{V}\right)^{2}} \sim \frac{1}{\left(T-T_{\mathrm{G}}\right)^{2 n-3}}, \quad \overline{\left(\delta \mu_{n} \sqrt{V}\right)^{2}} \sim \frac{1}{\left|\varphi-\varphi_{G}\right|^{2 n-3}}
$$

where the right and left expressions correspond to different protocols to induce the transition (cooling and compression). This 


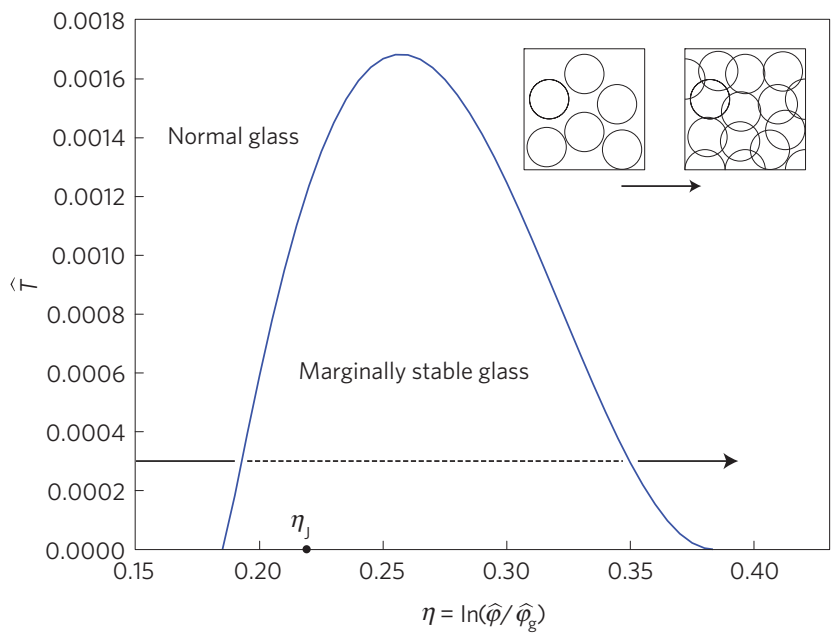

Figure 3 | Gardner critical packing fractions $\varphi_{G}$ obtained by compressing amorphous solids at fixed temperature. All amorphous solids are formed at the same $\widehat{\varphi}_{g}=8$. The location of the jamming point is denoted by $\eta_{\mathrm{J}}$.

increase leads to giant fluctuations at the Gardner transition. Finite-size (mean-field) scaling implies that, at the transition, $\overline{\left(\delta \mu_{n} \sqrt{V}\right)^{2}} \sim V^{2 n / 3-1}$. For $n=2$, fluctuations are subleadinghence the linear elastic shear modulus is regular and well defined: $\mu_{2} \simeq \bar{\mu}_{2}+O\left(V^{-1 / 6}\right)$. In contrast, all nonlinear moduli are not: their fluctuations diverge as $V^{n / 3-1}$ and completely overwhelm the average, which remains finite but is not representative of the typical behaviour. Note, moreover, that all odd moduli, which vanish by symmetry in ordered solids, can be neglected only above $T_{\mathrm{G}}$. At $T_{\mathrm{G}}$ they also blow up and, instead of being of the order of $1 / \sqrt{V}$ (and zero in average), they diverge as $V^{n / 3-1}$ and fluctuate. All these results signal that standard elastic behaviour breaks down at $T_{\mathrm{G}}$. Below $T_{\mathrm{G}}$, even an infinitesimal deformation leads in the thermodynamic limit to ageing and time-dependent shear moduli. In this regime, elastic moduli depend on the history and on the protocol used to measure them. Only strains whose amplitude scales to zero with $V$ do not lead to ageing and irreversible behaviour ${ }^{8}$. The elastic moduli computed in this way, called quenched in ref. 8 and zero-field cooled in ref. 33, are a property of the metabasin to which the system belongs. They are characterized by the same divergent fluctuations found at $T_{\mathrm{G}}$. This is a consequence of the marginal stability of glasses within the whole Gardner phase. We derived our results in a specific realistic model in the limit of infinite dimensions. However, our findings go beyond the specific $d \rightarrow \infty$ computation we have presented, and hold for generic interaction potentials. Indeed, one can obtain them using a Landau theory, as shown in the Supplementary Methods. Similar to the existence of diverging magnetic responses at a ferromagnetic transition, the breakdown of elastic behaviour and the divergence of nonlinear elastic moduli are the generic signature of the Gardner transition. Since our results are derived essentially using a Landau theory approach, they can be easily extended mutatis mutandis to spin glasses in a field and provide new ways to test for the existence of the de Almeida-Thouless line.

Our results reveal that the jerky elastic behaviour exhibited by amorphous solids at low temperature could be related to the existence of a phase transition within the glass phase. Our exact solution in the limit of infinite dimensions characterizes the dependence of this transition on the control parameters $(T, \varphi)$ and protocols (cooling, compression), providing guidance for experimental tests both in structural, colloidal, and possibly granular glasses. As has been shown in refs 30,31, the Gardner transition can be induced not only by decreasing the temperature or increasing the pressure, but also by straining an amorphous solid. We expect that our results generalize to this case: standard elastic behaviour has to break down at the finite value of the strain corresponding to the Gardner transition. To substantiate our predictions, it would be interesting to extensively study by experiments and by simulations whether standard elastic behaviour breaks down at a well-defined temperature in the way identified in this work. The simulations of model systems quenched at zero temperature by Procaccia et al. show very promising results: the linear elastic intra-state modulus is found to be well defined, whereas $\mu_{3}$ has fluctuations of $O(1)$ and $\mu_{4}$ shows diverging fluctuations, as we found. On the theoretical side, it is important to go beyond the Landau theory to obtain more quantitative predictions of the value of the critical exponents controlling the divergence of the elastic moduli ${ }^{34}$. Moreover, it is certainly worth exploring the implications of our results on the statistics of avalanches induced by strain, generalizing the ideas originally put forward in ref. 2, and connect our results with studies on shear-transformation-zone interactions $\mathrm{s}^{21}$.

All these factors open the way towards new research directions aimed at revealing the true nature of glasses. As suggested by several recent research results on jamming and amorphous plasticity, glasses might not be just liquids that have ceased to flow, but an entirely different new kind of solid ${ }^{10,35,36}$.

Data availability. The data that support the plots within this paper and other findings of this study are available from the corresponding author upon request.

Received 1 February 2016; accepted 30 June 2016; published online 8 August 2016

\section{References}

1. Barrat, J.-L. \& Lemartre, A. Dynamical Heterogeneities in Glasses, Colloids, and Granular Media (eds Berthier, L., Biroli, G., Bouchaud, J.-P., Cipelletti, L. \& van Saarloos, W.) (Oxford Univ. Press, 2011).

2. Combe, G. \& Roux, J.-N. Strain versus stress in a model granular material: a Devil's staircase. Phys. Rev. Lett. 85, 3628-3631 (2000).

3. Maloney, C. E. \& Lemaître, A. Amorphous systems in athermal, quasistatic shear. Phys. Rev. E 74, 016118 (2006)

4. Lemaître, A. \& Caroli, C. Rate-dependent avalanche size in athermally sheared amorphous solids. Phys. Rev. Lett. 103, 065501 (2009).

5. Karmakar, S., Lerner, E. \& Procaccia, I. Statistical physics of the yielding transition in amorphous solids. Phys. Rev. E 82, 055103 (2010).

6. Lin, J., Lerner, E., Rosso, A. \& Wyart, M. Scaling description of the yielding transition in soft amorphous solids at zero temperature. Proc. Natl Acad. Sci. USA 111, 14382-14387 (2014)

7. Hentschel, H. G. E., Karmakar, S., Lerner, E. \& Procaccia, I. Do atherma amorphous solids exist? Phys. Rev. E 83, 061101 (2011).

8. Dubey, A. K., Procaccia, I., Shor, C. \& Singh, M. Elasticity in amorphous solids: Nonlinear or piecewise linear? Phys. Rev. Lett. 116, 085502 (2016).

9. Liu, A. J., Nagel, S. R., Van Saarloos, W. \& Wyart, M. Dynamical Heterogeneities in Glasses, Colloids, and Granular Media (eds Berthier, L., Biroli, G., Bouchaud, J.-P., Cipelletti, L. \& van Saarloos, W.) (Oxford Univ. Press, 2011).

10. Charbonneau, P., Kurchan, J., Parisi, G., Urbani, P. \& Zamponi, F. Fractal free energies in structural glasses. Nature Commun. 5, 3725 (2014).

11. Kurchan, J., Parisi, G. \& Zamponi, F. Exact theory of dense amorphous hard spheres in high dimension. I. The free energy. J. Stat. Mech. 2012, P10012 (2012)

12. Kurchan, J., Parisi, G., Urbani, P. \& Zamponi, F. Exact theory of dense amorphous hard spheres in high dimension. II. The high density regime and the Gardner transition. J. Phys. Chem. B 117, 12979-12994 (2013).

13. Gardner, E. Spin glasses with p-spin interactions. Nucl. Phys. B 257, 747-765 (1985)

14. Gross, D. J., Kanter, I. \& Sompolinsky, H. Mean-field theory of the Potts glass. Phys. Rev. Lett. 55, 304-307 (1985).

15. Berthier, L. et al. Growing timescales and lengthscales characterizing vibrations of amorphous solids. Proc. Natl Acad. Sci. USA 113, 8397-8401 (2016).

16. O’Hern, C. S., Silbert, L. E., Liu, A. J. \& Nagel, S. R. Jamming at zero temperature and zero applied stress: the epitome of disorder. Phys. Rev. E 68, 011306 (2003).

17. Wyart, M., Nagel, S. R. \& Witten, T. A. Geometric origin of excess low-frequency vibrational modes in weakly connected amorphous solids. Europhys. Lett. 72, 486-492 (2005). 
18. Wyart, M. Marginal stability constrains force and pair distributions at random close packing. Phys. Rev. Lett. 109, 125502 (2012).

19. Lerner, E., DeGiuli, E., Düring, G. \& Wyart, M. Breakdown of continuum elasticity in amorphous solids. Soft Matter 10, 5085-5092 (2014).

20. DeGiuli, E., Laversanne-Finot, A., Düring, G., Lerner, E. \& Wyart, M. Effects of coordination and pressure on sound attenuation, boson peak and elasticity in amorphous solids. Soft Matter 10, 5628-5644 (2014).

21. Müller, M. \& Wyart, M. Marginal stability in structural, spin, and electron glasses. Annu. Rev. Condens. Matter Phys. 6, 177-200 (2015).

22. Franz, S., Parisi, G., Urbani, P. \& Zamponi, F. Universal spectrum of normal modes in low-temperature glasses. Proc. Natl Acad. Sci. USA 112, 14539-14544 (2015).

23. Berthier, L. \& Witten, T. A. Glass transition of dense fluids of hard and compressible spheres. Phys. Rev. E 80, 021502 (2009).

24. Schmiedeberg, M., Haxton, T. K., Nagel, S. R. \& Liu, A. J. Mapping the glassy dynamics of soft spheres onto hard-sphere behavior. Europhys. Lett. 96, 36010 (2011).

25. Berthier, L., Biroli, G., Coslovich, D., Kob, W. \& Toninelli, C. Finite-size effects in the dynamics of glass-forming liquids. Phys. Rev. E 86, 031502 (2012).

26. Debenedetti, P. G. \& Stillinger, F. H. Supercooled liquids and the glass transition. Nature 410, 259-267 (2001).

27. Franz, S. \& Parisi, G. Structural glass transition and the entropy of the metastable states. J. Phys. I 5, 1401-1415 (1995).

28. Monasson, R. Structural glass transition and the entropy of the metastable states. Phys. Rev. Lett. 75, 2847-2850 (1995).

29. Charbonneau, P., Kurchan, J., Parisi, G., Urbani, P. \& Zamponi, F. Exact theory of dense amorphous hard spheres in high dimension. III. The full replica symmetry breaking solution. J. Stat. Mech. 2014, P10009 (2014).

30. Rainone, C., Urbani, P., Yoshino, H. \& Zamponi, F. Following the evolution of hard sphere glasses in infinite dimensions under external perturbations: compression and shear strain. Phys. Rev. Lett. 114, 015701 (2015).
31. Rainone, C. \& Urbani, P. Following the evolution of glassy states under external perturbations: the full replica symmetry breaking solution. J. Stat. Mech. 2016, 053302 (2016)

32. Charbonneau, P., Corwin, E. I., Parisi, G., Poncet, A. \& Zamponi, F. Universal non-Debye scaling in the density of states of amorphous solids. Preprint at http://arXiv.org/abs/1512.09100 (2015)

33. Nakayama, D., Yoshino, H. \& Zamponi, F. Protocol-dependent shear modulus of amorphous solids. Preprint at http://arXiv.org/abs/1512.06544 (2015).

34. Urbani, P. \& Biroli, G. Gardner transition in finite dimensions. Phys. Rev. B 91 , $100202(2015)$

35. Goodrich, C. P., Liu, A. J. \& Nagel, S. R. Solids between the mechanical extremes of order and disorder. Nature Phys. 10, 578-581 (2014).

36. Biroli, G. Disordered solids: in search of the perfect glass. Nature Phys. 10, 555-556 (2014).

\section{Acknowledgements}

We thank J.-P. Bouchaud, P. Charbonneau, S. Franz, I. Procaccia, G. Tarjus, M. Wyart, $\mathrm{H}$. Yoshino and F. Zamponi for useful discussions. We acknowledge financial support from the ERC grant NPRGGLASS and from a grant of the Simons Foundation (\#454935, G.B.).

\section{Author contributions}

All authors contributed to all aspects of this work.

\section{Additional information}

Supplementary information is available in the online version of the paper. Reprints and permissions information is available online at www.nature.com/reprints.

Correspondence and requests for materials should be addressed to G.B.

\section{Competing financial interests}

The authors declare no competing financial interests. 